\title{
Attention aux propositions d'inscription dans un registre électronique
}

Service juridique de la FMH

Correspondance:

Service juridique de la FMH Elfenstrasse 18

Case postale 170

CH-3000 Berne 15

Tél. 0313591111

Fax 0313591112
Depuis l'affaire Printus Verlag AG, qui a pris de l'ampleur (cf. notre site internet www.fmh.ch $\rightarrow$ Nos prestations $\rightarrow$ Droit $\rightarrow$ Médecin et droit $\rightarrow$ Divers $\rightarrow$ Printus-Verlag SA: annuaire meinhaus arzt.ch), nous sommes régulièrement contactés par des membres qui nous informent avoir reçu une nouvelle «offre» pour une inscription dans un annuaire électronique. Très souvent, ces offres sont en réalité des combines visant à encaisser de l'argent et non à proposer un vrai service aux médecins.

Les médecins sont apparemment une cible privilégiée et de nouveaux formulaires / de nouvelles méthodes d'accrochage apparaissent sans cesse. Voici donc quelques conseils de prudence à observer:

1. Nous vous rappelons tout d'abord que la FMH tient un registre des médecins, public et gratuit, qui peut être consulté sur notre site internet.

2. Avant de vous inscrire dans un nouveau registre, demandez-vous donc si vous en avez réellement l'utilité.

3. Si vous souhaitez vous inscrire, renseignezvous sur ledit registre pour vous assurer qu'il ne s'agit pas d'une «arnaque»: existe-t-il réellement? Fonctionne-t-il bien? Quelle est la société qui l'exploite?

4. Faites attention au prix demandé: s'agit-il d'une somme mensuelle ou annuelle? Le prix est-il raisonnable par rapport à la prestation offerte?

5. Ce genre d'«offres» commence souvent par des phrases telles que «selon votre demande» ou «confirmation de votre qualité de membre». Cela donne l'impression qu'il s'agit de choses déjà commandées - donc déjà examinées et contrôlées - et qu'il suffit de signer et de payer. Ainsi, tout ce qui semble avoir déjà été commandé ne l'a pas forcément réellement été.

6. Ces offres se font sous plusieurs formes (téléphone, envoi d'un formulaire à remplir, envoi d'une lettre informative accompagnée d'une facture, etc.). Pour éviter qu'un(e) employé(e) ne signe un document par inadvertance, rendez votre personnel attentif à ce genre d'agissements.

A bon entendeur... 